\title{
BMR
}

\section{Development and characterization of microsatellite markers of the eastern keelback mullet (Liza affinis)}

\author{
L. Liu', T.X. Gao' ${ }^{2}$ Z.Q. Han ${ }^{2}$, C.H. Li ${ }^{3}$, D.R. Sun ${ }^{3}$ and N. Song ${ }^{1}$ \\ ${ }^{1}$ Fisheries College, Ocean University of China, Qingdao, China \\ ${ }^{2}$ Fishery College, Zhejiang Ocean University, Zhoushan, China \\ ${ }^{3}$ South China Sea Fisheries Research Institute, \\ Chinese Academy of Fishery Sciences, Guangzhou, China \\ Corresponding author: N. Song \\ E-mail: songna624@163.com
}

Genet. Mol. Res. 15 (2): gmr. 15028380

Received January 4, 2016

Accepted March 11, 2016

Published June 17, 2016

DOI http://dx.doi.org/10.4238/gmr.15028380

\begin{abstract}
Twenty-four polymorphic microsatellite loci were isolated and characterized for Liza affinis using a (GT)13-enriched genomic library. The number of alleles per locus ranged from 3 to 9 , with a mean number of 6.250 . The observed and expected heterozygosities ranged from 0.417 to 1.000 and from 0.550 to 0.861 , with an average of 0.859 and 0.779 , respectively. Deviation from Hardy-Weinberg proportions was detected at three loci. Evidence of null alleles was found at two loci. These markers will be useful in further studies investigating the genetic variation and population structure of this species, and may provide insights into the maintenance and efficient management of eastern keelback mullet resources.
\end{abstract}

Key words: Liza affinis; Microsatellite loci; Population structure; Genetic diversity 


\section{INTRODUCTION}

Eastern keelback mullet (Liza affinis) belongs to the family Mugilidae, and is widely distributed in Japan (except for northern Hokkaido) and along the coasts of China, from the Yellow Sea to the South China Sea (Senou et al., 1987). With the decline of many traditional economic fish resources, $L$. affinis is gradually becoming a new kind of important economic fish for the global fisheries industry. In order to prevent the reduction of $L$. affinis populations, it is important to characterize the population structure, which may provide insights into the maintenance and efficient management of $L$. affinis resources. Because of their high polymorphism, reproducibility, relative abundance, and multi-allelic and co-dominant nature, microsatellite loci are considered to be powerful genetic markers and have been widely used in population structure analyses. Here, for the first time, we isolated microsatellite loci for $L$. affinis, which will provide a powerful tool for analyzing population structure of $L$. affinis. A limitation of this study was that the number of microsatellite loci developed using streptavidin-coated magnetic beads was lower than the number developed using a nextgeneration sequencing platform.

\section{MATERIAL AND METHODS}

Twenty-four individuals were collected from coastal waters near Zhanjiang, China, and preserved at $-20^{\circ} \mathrm{C}$ until DNA extraction. Genomic DNA was extracted from muscle tissue of $L$. affinis using the standard phenol-chloroform method. A microsatellite-enriched genomic library was constructed following the enrichment protocols described by Ma and Chen (2009). Genomic DNA was digested with MseI restriction enzyme (New England Biolabs, USA). The adapters (5'-TAC TCA GGA CTC AT-3'/5'-GAC GAT GAG TCC TGA G-3') were ligated to the DNA fragments. The ligated products were then pre-amplified in a $25-\mu \mathrm{L}$ reaction system using an adapter specific primer (5'-GAT GAG TCC TGA GTA A-3') to verify the ligation and to increase the DNA concentration (Lin et al., 2015). Subsequently, the ligation products were hybridized to the biotin-labeled probe, (GT)13. Next, fragments containing simple repeat sequences were captured by streptavidin-coated magnetic beads (Promega, USA), washed, and recovered by heating and ethanol precipitation. The enriched fragments were amplified by polymerase chain reaction (PCR) using the adapter specific primer. The final amplification products were ligated into the pMD 18-T vectors (TaKaRa, Japan) and transformed into Escherichia coli DH5 $\alpha$ competent cells. A total of 142 positive clones were selected using blue/white screening, and were sequenced by an ABI Prism 3730 automated DNA Analyzer (Applied Biosystems, USA). Following sequence analysis, PCR primer pairs were designed to amplify 119 microsatellite loci with suitable flanking regions using the PRIMER PREMIER 5 software (Premier Biosoft International, USA).

The designed primers were analyzed using 24 L. affinis individuals from the coastal waters near Zhanjiang, China. The PCR was carried out in a $25-\mu \mathrm{L}$ reaction mixture containing $17.25 \mu \mathrm{L}$ ultrapure water, $2.5 \mu \mathrm{L} \mathrm{10X} \mathrm{PCR} \mathrm{buffer,} 2 \mu \mathrm{L}$ dNTPs, $1 \mu \mathrm{L}$ each primer $(5 \mu \mathrm{M})$, $0.25 \mu \mathrm{L}$ Taq polymerase, and $1 \mu \mathrm{L}$ DNA template. The PCR program consisted of an initial step for $2 \mathrm{~min}$ at $95^{\circ} \mathrm{C}$ followed by 35 cycles of $0.5 \mathrm{~min}$ at $94^{\circ} \mathrm{C}, 1 \mathrm{~min}$ at the locus-specific annealing temperature (Table 1), and $1 \mathrm{~min}$ at $72^{\circ} \mathrm{C}$, and a final step for $10 \mathrm{~min}$ at $72^{\circ} \mathrm{C}$, after which the reaction was held at $4^{\circ} \mathrm{C}$. Negative controls were included in all PCR amplifications 
to confirm the absence of contaminants. PCR products were purified with a Gel Extraction Mini Kit (Watson BioTechnologies Inc., China), separated on 6\% denaturing polyacrylamide gel, and visualized by silver staining (Lin et al., 2011).

Table 1. Characteristics of microsatellite loci in Liza affinis.

\begin{tabular}{|c|c|c|c|c|c|c|c|c|}
\hline Locus name & Primer sequence $\left(5^{\prime}-3^{\prime}\right)$ & Repeat motif & $\mathrm{Ta}\left({ }^{\circ} \mathrm{C}\right)$ & Allele size range (bp) & Sample size & $N_{\mathrm{A}}$ & $H_{\mathrm{O}}$ & $H_{\mathrm{E}}$ \\
\hline La77 & $\begin{array}{l}\text { F: AAAGGGCACAAGGTGAGTAAA } \\
\text { R: GCGTCCCATATCTGAGCAGT }\end{array}$ & $(\mathrm{AC})_{10}$ & 52 & $240-280$ & 24 & 5 & 1.000 & 0.789 \\
\hline La79 & $\begin{array}{l}\text { F: AGACGGGAAAGGAGTGGAAGA } \\
\text { R: CCAGGGCACAGCATATTGAGA }\end{array}$ & $(\mathrm{TG})_{8} \ldots(\mathrm{TG})_{8}$ & 49 & $220-270$ & 24 & 6 & 0.999 & 0.826 \\
\hline La83 & $\begin{array}{l}\text { F: CTGTCCCCTGCTGACCTAA } \\
\text { R: GTTACCAAACATTAAAAGCCAT }\end{array}$ & $(\mathrm{TG}) 15$ & 48 & $254-380$ & 24 & 6 & 1.000 & 0.803 \\
\hline La89 & $\begin{array}{l}\text { F: ATCACATCTACCTCCAGT } \\
\text { R: ATTTCCATACAGTCTAATG }\end{array}$ & (TG)9 & 48 & $208-232$ & 24 & 3 & 0.667 & 0.582 \\
\hline La106 & $\begin{array}{l}\text { F: TAAGGGATGGGCTGTATTTG } \\
\text { R: CATTGATGGCATTATCATTAATA }\end{array}$ & $(\mathrm{AC})_{14}$ & 55 & $220-320$ & 24 & 8 & 1.000 & 0.861 \\
\hline La112 & $\begin{array}{l}\text { F: CTGGATCATGGGGCTTAC } \\
\text { R: TACTGGCTGCTGCCTTCT }\end{array}$ & $\begin{array}{l}(\mathrm{TC})_{9} \ldots(\mathrm{TC})_{7} \ldots \\
(\mathrm{CA})_{6} \ldots(\mathrm{CA})_{7}\end{array}$ & 58 & $220-240$ & 24 & 7 & 0.667 & 0.782 \\
\hline La138* & $\begin{array}{l}\text { F: ATTGTTCATACCGTAGTGC } \\
\text { R: GATTAAGCCAAGAAGAGG }\end{array}$ & $(\text { CTCCT })_{5}$ & 51 & $201-242$ & 24 & 7 & 0.500 & 0.793 \\
\hline $\mathrm{La} 139^{* \dagger}$ & $\begin{array}{l}\text { F: CTTTATGTCTCCTCCAGCGTT } \\
\text { R: TGTGCCTTTTCTAATGTCAGC }\end{array}$ & $(\mathrm{CTCCT})_{5} \ldots(\mathrm{GT})_{5}$ & 60 & $210-240$ & 24 & 5 & 0.417 & 0.794 \\
\hline La140 & $\begin{array}{l}\text { F: CACTCCACCATACACCTCC } \\
\text { R: CACTGGCTGCTTTACCTTA }\end{array}$ & $(\mathrm{AC})_{34}$ & 51 & $210-250$ & 24 & 8 & 1.000 & 0.806 \\
\hline La153 & $\begin{array}{l}\text { F: GGCTTTACTTAATTGTTTTGTTC } \\
\text { R: GCTTCACTTTCTCAGGCTCT }\end{array}$ & $\begin{array}{c}\mathrm{TG})_{5} \ldots(\mathrm{GA})_{6} \ldots \\
(\mathrm{AG})_{5} \ldots(\mathrm{GA})_{6}\end{array}$ & 51 & $200-230$ & 24 & 3 & 0.833 & 0.550 \\
\hline La165 & $\begin{array}{l}\text { F: CAGTTTGTGCTTTTAGGT } \\
\text { R: CTTTTGTAGGAGCGTTAT }\end{array}$ & $(\mathrm{GA})_{7 \ldots}(\mathrm{GA})_{18}$ & 51 & $280-380$ & 24 & 9 & 1.000 & 0.828 \\
\hline La170 & $\begin{array}{l}\text { F: TCTCCTCCTACGGGTCAG } \\
\text { R: CAGCCAAACGATCACAAA }\end{array}$ & $(\mathrm{TG})_{12}$ & 55 & $205-240$ & 24 & 8 & 1.000 & 0.840 \\
\hline La172 & $\begin{array}{l}\text { F: CACTGGTAAATTCCTTTCAGA } \\
\text { R: CGTTACATACGACGCATACA }\end{array}$ & $(\mathrm{CA})_{10}$ & 55 & $180-220$ & 24 & 5 & 0.833 & 0.796 \\
\hline La173 & $\begin{array}{l}\text { F: TAGACTGCCGCTAATTTCACT } \\
\text { R: ACGAGGAGACTTATCCCTGTA }\end{array}$ & $(\mathrm{CA})_{9} \ldots(\mathrm{CA})_{17}$ & 56 & $220-280$ & 24 & 8 & 1.000 & 0.849 \\
\hline La188 ${ }^{\dagger}$ & $\begin{array}{l}\text { F: TTGCCAAATTGCTAGATC } \\
\text { R: GGAGGAATAGTGGGAGAA }\end{array}$ & $(\mathrm{AC})_{10}$ & 48 & $210-240$ & 24 & 6 & 0.958 & 0.789 \\
\hline La195 & $\begin{array}{l}\text { F: ACTATTCCCGAGGAGTCT } \\
\text { R: TTCCAGAATTTTGTCTTAAC }\end{array}$ & $(\mathrm{CA})_{5} \ldots(\mathrm{CA})_{17} \ldots(\mathrm{CA})_{5}$ & 48 & $190-250$ & 24 & 7 & 0.875 & 0.824 \\
\hline La200 & $\begin{array}{l}\text { F: TCCCTGACAGAATACCAAC } \\
\text { R: GGTGTAGTAGAGCGAGGC }\end{array}$ & $(\mathrm{GT}) 11$ & 50 & $240-270$ & 24 & 7 & 0.958 & 0.785 \\
\hline $\mathrm{La} 201$ & $\begin{array}{l}\text { F: GGTTAGGGTTGCTTTATC } \\
\text { R: GGTGTAGTAGAGCGAGGC }\end{array}$ & $(\mathrm{TG})_{5} \ldots(\mathrm{GT})_{11}$ & 51 & $280-320$ & 24 & 8 & 0.833 & 0.848 \\
\hline La214 & $\begin{array}{l}\text { F: GAATCATACATCCACCAGC } \\
\text { R: AACCCTAAACCCGTTCTC }\end{array}$ & (TG)11 & 48 & $220-270$ & 24 & 5 & 0.667 & 0.670 \\
\hline La217 & $\begin{array}{l}\text { F: ATCAGTCATTATTGCCTTTT } \\
\text { R: ACACGCATGAGTAAACTACAT }\end{array}$ & $(\mathrm{AC})_{6} \ldots(\mathrm{TG})_{8}$ & 48 & $250-300$ & 24 & 8 & 0.870 & 0.854 \\
\hline $\mathrm{La} 227$ & $\begin{array}{l}\text { F: CATCCCACATCACCAAAG } \\
\text { R: GATTACTAAAGGCTACTGCTAC }\end{array}$ & $(\mathrm{AC})_{10}$ & 53 & $200-320$ & 24 & 5 & 0.792 & 0.785 \\
\hline La241 & $\begin{array}{l}\text { F: GAGTTTGGTATTTATCTTGT } \\
\text { R: ATGCACGTTCATTTTAGT }\end{array}$ & $(\mathrm{TG})_{27}$ & 54 & $210-245$ & 24 & 5 & 0.792 & 0.691 \\
\hline $\mathrm{La} 251$ & $\begin{array}{l}\text { F: AGACCCTCAGCACCAACG } \\
\text { R: TCCGCCTATTGACTGTAGTTTT }\end{array}$ & $(\mathrm{CA})_{14}$ & 54 & $220-285$ & 24 & 6 & 0.958 & 0.816 \\
\hline $\mathrm{La}_{261^{\dagger}}$ & $\begin{array}{l}\text { F: ACTGAGGCTTGTGAATGG } \\
\text { R: CTATCAGGGAAGGGTTGG }\end{array}$ & (TG) 10 & 55 & $230-305$ & 24 & 5 & 1.000 & 0.740 \\
\hline
\end{tabular}

$\mathrm{Ta}=$ optimized annealing temperature; $N_{\mathrm{A}}=$ number of alleles; $H_{\mathrm{O}}=$ observed heterozygosity; $H_{\mathrm{E}}=$ expected heterozygosity. *Locus may harbor null alleles (null allele frequency $>5 \%$ ). 'Deviation from Hardy-Weinberg proportions (adjusted $\mathrm{P}$ value $<0.0021$ ).

The observed and expected heterozygosities and Hardy-Weinberg disequilibrium were calculated by GENEPOP 4.0 (Rousset, 2008). Null allele frequencies (Brookfield, 1996) were calculated by MICRO-CHECKER 2.2.3 (Van Oosterhout et al., 2004). All results for multiple tests were corrected using Bonferroni's correction (Rice, 1989).

\section{RESULTS}

Twenty-four out of 44 loci were clearly amplified and shown to be polymorphic. The number of alleles per locus ranged from 3 to 9 , with an average of 6.250 . The observed and expected heterozygosities ranged from 0.417 to 1.000 and from 0.550 to 0.861 , respectively, 
with an average of 0.859 and 0.779 (Table 1). Three loci showed significant deviation from Hardy-Weinberg proportions, except for the loci La 139, La 188, and La 261 after Bonferroni correction $(\mathrm{P}<0.0021)$. Two loci (La 138, La 139) showed evidence of null alleles (null allele frequency $>5 \%$ ). No genetic disequilibrium was detected in each locus pair.

\section{DISCUSSION}

According to the results showed above, these 24 new microsatellite markers could be used to analyze the genetic structure of $L$. affinis and to generate important genetic data for the conservation and recovery of $L$. affinis resources.

\section{ACKNOWLEDGMENTS}

Research supported by the Special Fund for Agroscientific Research in the Public Interest (\#201303048) and the National Natural Science Foundation of China (\#41506158). This study was also supported by the Scientific Research Starting Foundation of Zhejiang Ocean University.

\section{REFERENCES}

Brookfield JFY (1996). A simple new method for estimating null allele frequency from heterozygote deficiency. Mol. Ecol. 5: 453-455. http://dx.doi.org/10.1111/j.1365-294X.1996.tb00336.x

Lin L, Zhu L, Liu SF, Su YQ, et al. (2011). Polymorphic microsatellite loci for the Japanese anchovy Engraulis japonicus (Engraulidae). Genet. Mol. Res. 10: 764-768. http://dx.doi.org/10.4238/vol10-2gmr1085

Lin L, Li CH, Xu SN, Liu Y, et al. (2015). Isolation and characterization of novel polymorphic microsatellite markers for Lutjanus erythropterus. Genet. Mol. Res. 14: 10944-10947. http://dx.doi.org/10.4238/2015.September.21.5

Ma HY and Chen SL (2009). Isolation and characterization of 31 polymorphic microsatellite markers in barfin flounder (Verasper moseri) and the cross-species amplification in spotted halibut (Verasper variegatus). Conserv. Genet. 10: 1591-1595. http://dx.doi.org/10.1007/s10592-008-9801-0

Rice WR (1989). Analyzing tables of statistical tests. Evolution 43: 223-225. http://dx.doi.org/10.2307/2409177

Rousset F (2008). genepop'007: a complete re-implementation of the genepop software for Windows and Linux. Mol. Ecol. Resour. 8: 103-106. http://dx.doi.org/10.1111/j.1471-8286.2007.01931.x

Senou H, Yoshino T and Okiyama M (1987). A review of the mullets with a keel on the back, Liza carinata complex (Pisces:Mugilide). Publ. Seto Mar. Biol. Lab. 32: 303-321.

Van Oosterhout C, Hutchinson WF, Wills DP and Shipley P (2004). MICRO-CHECKER: software for identifying and correcting genotyping errors in microsatellite data. Mol. Ecol. Notes 4: 535-538. http://dx.doi.org/10.1111/j.1471$\underline{8286.2004 .00684 . \mathrm{x}}$ 